\title{
Forschungsdatenmanagement und Informationskompetenz - Neue Entwicklungen an Hochschulbibliotheken Neuseelands
}

DOI 10.1515/iwp-2015-0040

Die Beschäftigung mit Forschungsdaten als Grundlage der wissenschaftlichen Arbeit ist ein neues Feld für Bibliotheken. Ihre Rolle in diesem Bereich und die Services, die sie für Wissenschaftlerinnen und Wissenschaftler entwickeln könnten, wird gerade verhandelt und gewinnt vor dem Hintergrund veränderter digitaler wissenschaftlicher Arbeitsrealität an Bedeutung. Der Begriff Informationskompetenz hat hierbei im anglo-amerikanischen Raum eine Ausweitung erfahren, der den gesamten Forschungsprozess inklusive des Umgangs mit Forschungsdaten beschreibt und mittlerweile auch in Deutschland aufgegriffen wird. Nach einer kurzen Erläuterung zur Weiterentwicklung des Begriffs Informationskompetenz wird untersucht, welche Services und Strukturen es in Neuseeland im Bereich Forschungsdaten bereits gibt und welche Faktoren und Probleme den Aufbau von Services beeinflussen. Neuseeland kann mit seinem überschaubaren, aber gut entwickelten Hochschulsystem genutzt werden, um den Blick für ähnliche Problemstellungen in Deutschland zu schärfen. Gleichzeitig wird mit dem Ansatz der Research Content Ecology der Universität Lincoln ein holistischer Ansatz analysiert, der konzeptionell und strukturell einen umfassenden Service im Bereich der Forschungsunterstützung und Informationskompetenz ermöglicht und als Anregung für den deutschen Kontext dienen kann.

Deskriptoren: Forschung, Informationskompetenz, Bibliothek, Dienstleistung, Neuseeland

Research Data Management and Information Literacy New Developments at New Zealand University Libraries Dealing with research data as the basis of academic research is a new field for libraries. Their role and scope in this new field is still being discussed and gains importance within the changing reality of the digital academic research setting. In Anglo-American countries as well as in Germany, the concept of information literacy has been broadened and now includes the whole academic research process as well as dealing with research data. After a short glance at the broadened concept of information literacy, the author analyses which services and structures are being developed and applied in New Zealand in the field of research data management and reflects on the problems which have to be considered when setting up these new services. New Zealand with its welldeveloped tertiary education system can serve as a model here to see which problems have to be taken into account when striving for the same aim in Germany. Structurally and conceptually, the holistic Research Content Ecology model of Lincoln University can serve as an example for the German context on how to establish a university-wide service for the support of research activities and the enhancement of researchers' information literacy.

Descriptors: Research, Data, Information literacy, Library, Service, New Zealand

La gestion des données de recherche et la maîtrise de l'information - de nouveaux développements dans les bibliothèques universitaires en Nouvelle-Zélande

L'étude des données de recherche comme base du travail scientifique est un nouveau domaine pour les bibliothèques. Le rôle de ces dernières dans ce domaine et les services qu'elles pourraient développer pour les scientifiques est en cours de négociation et gagne en importance sur fond de changements de la réalité de travail scientifique numérique. Le terme "compétence de l'information» qui décrit le processus de recherche, y compris le traitement des données de recherche, a connu une expansion dans le monde anglo-américain et est désormais également relayé en Allemagne. Après une brève introduction au développement du concept compétence de l'information, l'article examine les services et structures 
qui existent déjà en Nouvelle-Zélande dans le domaine des données de recherche, ainsi que les facteurs et problèmes qui influencent le développement de services. La Nouvelle-Zélande avec son système d'enseignement supérieur gérable, mais bien développé, peut être utilisée pour aiguiser notre regard sur des problèmes similaires en Allemagne. En même temps, l'auteur analyse la Research Content Ecology, une approche holistique de l'Université de Lincoln qui permet un service complet au niveau conceptuel et structurel dans le domaine de l'appui de la recherche et de la compétence de l'information, et qui peut servir d'inspiration dans le contexte allemand.

Descripteurs: recherche, faits, compétence de l'information, librairie, service d'information, Nouvelle-Zélande

\section{Einleitung}

Das Thema Forschungsdatenmanagement ist in aller Munde. Neue Services für Forschende sollen etabliert werden, neben der Ablage und Langzeitverfügbarkeit von Daten stehen auch Fragen der Lizenzierung, der Zitierbarkeit und der Sicherheit von Daten auf der Agenda. Zudem scheint immer wieder das Thema Open Data, also das freie Teilen von Forschungsdaten, am Horizont auf sicherlich ein hoher Anspruch, der sich mit der Realität im wissenschaftlichen Alltag einiger Disziplinen derzeit noch nicht vereinbaren lässt. ${ }^{1}$

Ende 2012 hat die Hochschulrektorenkonferenz in einer Empfehlung zudem den Umgang mit Forschungsdaten $\mathrm{zu}$ einem wichtigen Teilbereich der Informationskompetenz erklärt. Informationskompetenz umfasst demnach „[...], die Gesamtheit aller Fähigkeiten und Fertigkeiten, die erforderlich sind, um situationsrelevante Informationsbedarfe festzustellen, Information $\mathrm{zu}$ beschaffen, weiterzuverarbeiten, zu bewerten, zu präsentieren und Nutzungsbedingungen von Information einzuordnen.' [...] Außerdem muss betont werden, dass Informationskompetenz heute in besonderer Weise auf den Umgang mit den neuen Entwicklungen der wissenschaftlichen Informationsinfrastrukturen (z. B. mit Virtuellen Forschungsumgebungen und Forschungsdaten) zu beziehen ist." (Hochschulrektorenkonferenz 2012, S. 6).

Was genau bedeutet dies aber für Ausbildung des akademischen Nachwuchses? Sollen und können sich

1 Vgl. Büttner/Hobohm/Müller 2011; Tappenbeck 2015; Pryor 2012 wissenschaftliche Bibliotheken hier engagieren und Informationskompetenz-Angebote entsprechend ausweiten? Wie wäre ein solches Angebot organisatorisch und inhaltlich umzusetzen?

Mit diesen Fragen im Kopf bin ich Anfang 2013 nach Neuseeland geflogen. Neuseeland ist nämlich nicht nur ein interessantes Land, sondern hat auch ein gut entwickeltes Hochschulsystem und eine lange Tradition im Bereich Informationskompetenz, wie sich nicht zuletzt an den ANZIL-Standards zeigt (s. Bundy 2004). Gute Voraussetzungen also, um hier Feldforschung zu betreiben und von dieser Perspektive aus einen Blick zurück nach Deutschland zu werfen.

\section{Überblick zur Fachdebatte}

Die oben genannten Fragen waren natürlich nicht der einzige Ausgangspunkt für die Forschungsreise nach Neuseeland. In der Fachdebatte gibt es einige interessante Aufsätze und Monographien, die sich gerade mit der Verknüpfung von Forschungsdatenmanagement und Informationskompetenz befassen. Hier im Folgenden nur ein kurzer Überblick - weitere Hinweise finden sich in der Masterarbeit (Steiner 2013, insbes. S. 7-30).

Früher als in Deutschland hat im anglo-amerikanischen Bereich der Begriff information literacy eine Erweiterung erfahren. So fasst die Definition der britischen Society of College, National and University Libraries dies folgendermaßen: „Information literacy is an umbrella term which encompasses concepts such as digital, visual and media literacies, academic literacy, information handling, information skills, data curation and data management.“ (SCONUL 2011, S. 3)

Neben dieser abstrakten Definition sind noch SCONULs Seven Pillars of Information Literacy mit dem Fokus Forschung von Bedeutung (vgl. Corrall 2012, S. 115), ebenso wie die Information Literacy Lens on the Vitae Researcher Development Framework (vgl. Vitae 2012). Beide Modelle fassen Informationskompetenz für Forschungsdatenmanagement jedoch sehr abstrakt. Zudem lässt sich feststellen, dass es noch keine Bezeichnung hierfür gibt, die einheitlich gebraucht wird. Hilfreich sind jedoch die etwas konkreter gefassten Kompetenzen, die Carlson et al. herauskristallisiert haben. Sie nutzen für diese Kernkompetenzen die Bezeichnung data information literacy (vgl. Carlson et al. 2013, S. 213):

- Datenbanken und Datenformate (Databases and Data Formats)

- Finden und Erwerben von Daten (Discovery and Acquisition) 
- Management und Organisation von Daten (Data Management and Organization)

- Konvertierung von Daten und Interoperabilität (Data Conversion and Interoperability)

- Datenqualität und -dokumentation (Data Quality and Documentation)

- Metadaten und Datenbeschreibung (Metadata and Data Description)

- Datenpflege und Nachnutzung (Data Curation and Reuse)

- Richtlinien zum adäquaten Umgang mit Daten (Cultures of Practice)

- Erhaltung von Daten (Data Preservation)

- Verarbeitung und Analyse von Daten (Data Processing and Analysis)

- Visualisierung und Darstellung von Daten (Data Visualization and Representation)

- Ethische Aspekte und ihre Anwendung, inklusive des Zitierens von Daten (Ethics and Attribution)

Deutlich wird hier ebenfalls, dass die fachliche Spezifizierung dieser Aspekte wichtig ist, damit sie für die Zielgruppen der verschiedenen Disziplinen tatsächlich handhabbar werden und von den Forschenden einsetzbar sind.

Neuseeland ist im Hinblick auf Aktivitäten von Hochschulbibliotheken im Forschungsdatenmanagement bisher nur selten untersucht worden. Maßgeblich sind hier die beiden Studien von Brown (2009) und Corrall et al. (2013). Beide zeigen deutlich, dass das Thema Forschungsdatenmanagement in Neuseeland noch in den Kinderschuhen steckt und Neuseeland damit im Vergleich zu Ländern wie Australien, den USA oder Großbritannien zurückliegt.

\section{Methodik}

Daher entschied ich mich, für die Masterarbeit qualitative Experteninterviews an möglichst allen Universitätsbibliotheken zu führen. Auf Grundlage der in der Literatur diskutierten Themen enthielt der Leitfaden folgende Themen: Bereits bestehende Services im Forschungsdatenmanagement, Zielgruppen dieser Services, der interne Blick der Bibliothek auf das neue Betätigungsfeld und die neue Rolle sowie der Blick der Universität auf die Rolle der Bibliothek. Sechs von acht Universitätsbibliotheken sagten einen Interviewtermin zu. Interviewt wurden die für Forschungsservices zuständigen Personen in der Bibliothek im Zeitraum März bis April 2013. Die Interviews wurden digital aufgezeichnet, transkribiert und in Anlehnung an die Grounded Theory ausgewertet. Bei der Auswertung wurden neben dem Servicespektrum der Bibliotheken im Bereich Forschungsdaten (und in data information literacy) auch untersucht, welche Hauptprobleme für die Entwicklung von Services die Interviewten sehen, die möglicherweise übertragbar sind auf deutsche Verhältnisse. Schließlich wurde analysiert, ob es strukturelle Ansätze gibt, die in Deutschland in ähnlicher Weise fruchtbar gemacht werden könnten.

\section{Ergebnisse}

Wie Abbildung 1 zeigt, boten die befragten Universitätsbibliotheken Services in sechs Bereichen an. Drei von sechs Bibliotheken unterstützten Forschende beispielsweise mit Hinweisen zur Ablage der Daten in Repositorien. Abzugrenzen davon sind längerfristige Beratungsangebote wie sie von zwei Bibliotheken geleistet wurden. Zwei Bibliotheken schließlich betrieben Repositorien zur Ablage von Daten von Masterarbeiten oder Dissertationen.

Befragt nach Problemstellungen, sahen die Interviewten das größte Hindernis in der Rolle der Forschenden selbst. Hier wurde nicht nur deutlich, dass die Interessen und Bedarfe der Forschenden je nach Fachrichtung sehr heterogen waren, sondern auch, dass die Bereitschaft zum Teilen von Daten sehr unterschiedlich ausgeprägt war. An fünf von sechs befragten Universitätsbibliotheken in Neuseeland war Forschungsdatenmanagement 2013 zudem noch kein Thema auf der Ebene der Universität, und vier Befragte betonten, dass es der nationalen Förderpolitik noch nicht gelungen sei, die Forschenden tatsächlich zum Teilen von Daten zu bewegen, wenn keine Persönlichkeitsrechte o. ä. entgegenstünde. Zudem wurde darauf hingewiesen, dass technologische Fragestellungen noch zu lösen seien und dass die Debatte zum Teilen von Daten und dem freien Zugang und der Nachnutzung von Daten (Shared Data/Open Data) noch nicht sehr weit fortgeschritten sei. Schließlich wurde in zwei Interviews herausgestellt, dass die neuseeländischen Universitäten und damit auch ihre Bibliotheken in starkem Wettbewerb um Fördermittel und Studierendenbeiträge stünden, so dass eine Zusammenarbeit der Universitätsbibliotheken sehr erschwert werde.

Zusammenfassend lässt sich die Situation einer Universitätsbibliothek wie in Grafik 3 darstellen. Idealerweise sind die Aufgaben im Forschungsdatenmanagement auf den verschiedenen Ebenen zufrieden stellend geklärt. Auf nationaler Ebene gibt es eine Förderpolitik, die Open Data propagiert und fördert, auf universitärer 


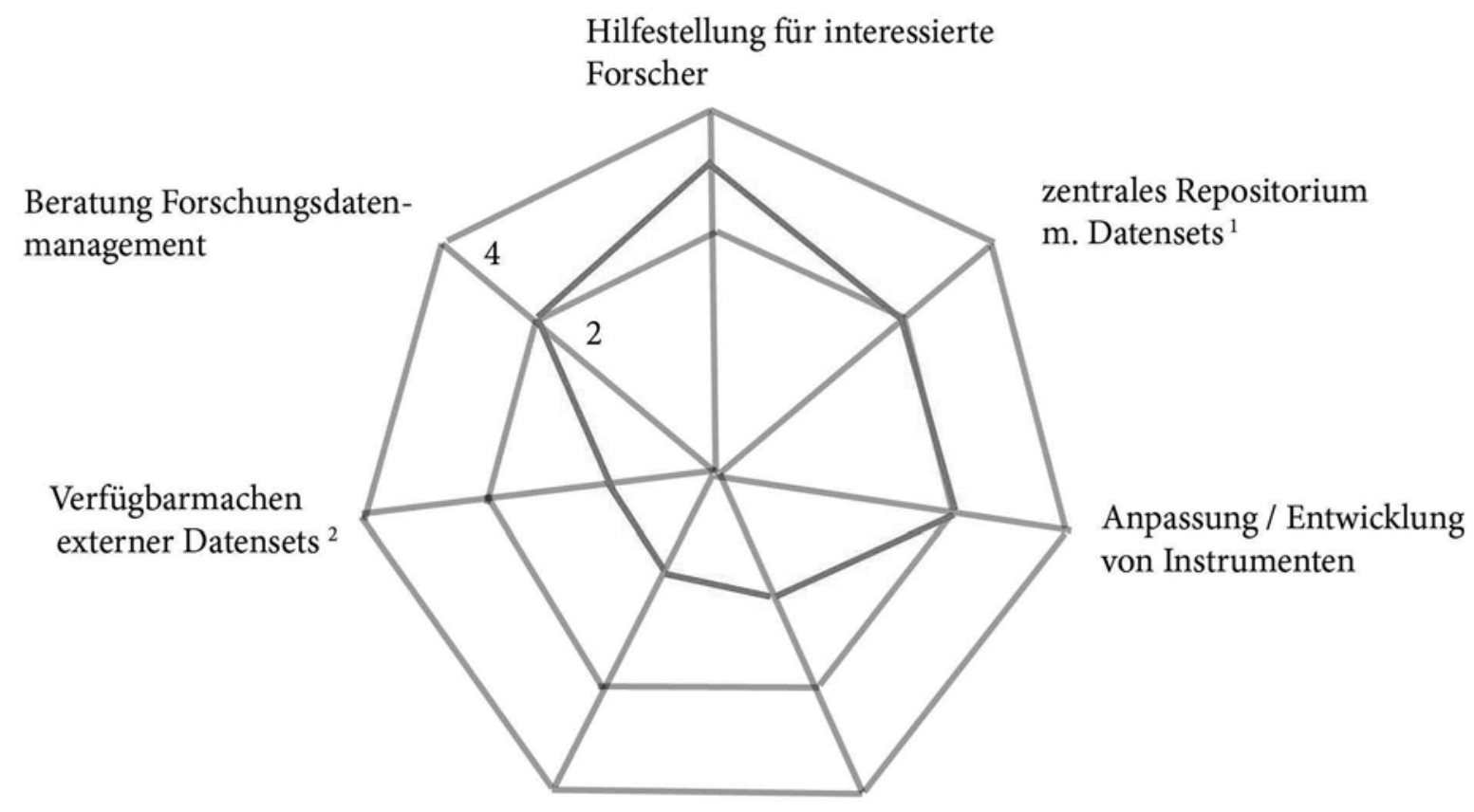

externe Datenablage

institutional policy vorhanden

$$
\mathrm{n}=6
$$

${ }^{1}$ wird mit wiss. Publikation abgelegt

${ }^{2}$ ggf. häufiger (wurde nur einmal explizit erwähnt)

Abbildung 1: Bestehende Services für Forschungsdaten an sechs von acht Universitätsbibliotheken Neuseelands im März/April 2013 (eigene Darstellung).

Rolle der Forscher (unterschiedliche Bedarfslagen, unterschiedliche Bereitschaft zum Teilen) nationale Zusammenarbeit oder Konkurrenz?

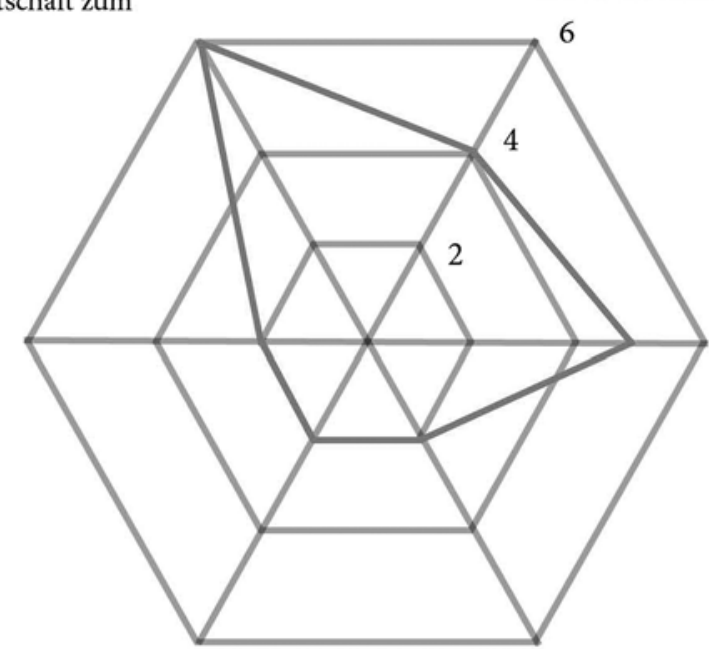

keine Auflagen

bei der Forschungsförderung
Forschungsdatenmanagement kein hochschulweites Thema
Paradigmenwechsel Open Data / Shared Data technologische Fragestellungen

$$
\mathrm{n}=6
$$

Abbildung 2: Überblick über Probleme für Forschungsdaten-Services nach Aussagen in den Interviews (März/April 2013) an sechs Universitätsbibliotheken in Neuseeland (eigene Darstellung). 
traditionelle wissenschaftliche Reputationsstruktur

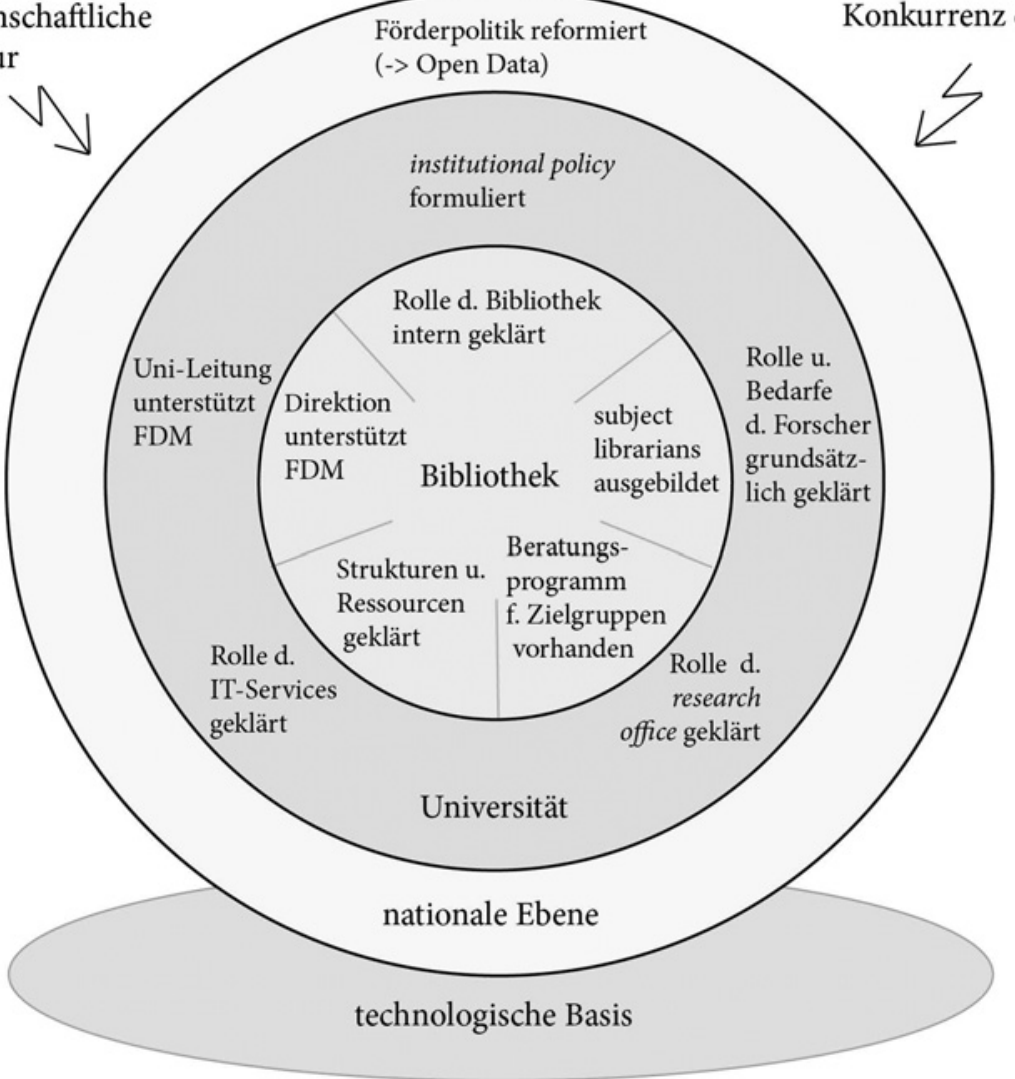

Abbildung 3: Problemfaktoren im Forschungsdatenmanagement aus Sicht einer Bibliothek (eigene Darstellung).

Ebene übernehmen die verschiedenen Institutionen (research office, university board, library, IT services) klar definierte Aufgaben und es gibt eine institutional policy, die auf Open Data zielt, und auch in der Bibliothek sind die Aufgaben, organisatorischen Strukturen und Ressourcen vorhanden, um effektive Services im Forschungsdatenmanagement bieten zu können. Hier wurden die subject librarians („Fachreferent*innen“) in mehreren Interviews als wichtige Ansprechpartner*innen für die Fachbereiche hervorgehoben. Ihrer Aus- und Weiterbildung kommt damit eine wichtige Rolle zu. Schließlich ist festzuhalten, dass eine verlässliche und einfach zugängliche technologische Grundlage wichtig ist. Weitere externe Faktoren können den Aufbau von Services im Forschungsdatenmanagement erheblich erschweren. In Neuseeland sind dies vor allem die Konkurrenzsituation der Universitäten untereinander sowie die traditionelle akademische Reputationsstruktur, die sich auf Veröffentlichungen stützt. Die verschiedenen Bereiche beeinflussen und bedingen einander, und es wird deutlich, dass verschiedene Bedingungen erfüllt sein müssen, um erfolgreich Services im Bereich Forschungsdatenmanage- ment anbieten zu können. Dies betrifft auch data information literacy-Services.

Als wichtiges Ergebnis auf organisatorischer Ebene lässt sich aus Neuseeland insbesondere das Modell der Lincoln University in der Nähe von Christchurch als interessante Referenz anführen, das für Universitäten in Deutschland möglicherweise gewinnbringend adaptiert werden könnte. In Lincoln bietet ein interdisziplinäres Team (Library, Teaching and Learning $=$ LTL) aus Forschenden, Lehrenden, Bibliothekar*innen und einer Psychotherapeutin Services im Bereich Informationskompetenz an. Von den Fachrichtungen her sind die Naturwissenschaften ebenso vertreten wie Geisteswissenschaften. LTL arbeitet eng mit anderen universitären Institutionen wie dem research office zusammen und zielt mit den Angeboten auf den gesamten Universitätszusammenhang. Verschiedene technologische Services wurden und werden unter dem Dach einer Research Content Ecology zusammengeführt (Dawson 2012). Theoretisch knüpft Lincoln dabei an das umfassende Konzept der information ecology von Nardi und O’Day an. Diese betonen, dass die Interaktion der Menschen innerhalb eines 
Systems wichtig ist und die technologischen Instrumente daran gemessen werden sollten, wie effektiv sie die Kommunikation und Kollaboration der Akteurinnen und Akteure untereinander befördern. (Nardi/O’Day 1999, hier S. 66)

LTL begreift Lernen und Forschen als ganzheitlichen Prozess, bei dem die Studierenden und Forschenden zu jeder Zeit möglichst umfassend unterstützt werden sollten und in dessen Verlauf auch Zwischenergebnisse veröffentlicht werden sollen, um weitere Forschungsfragen zu fördern. Daher gibt es neben dem Repositorium für Veröffentlichungen auch das Lincoln Community Archive, in dem Material abgelegt wird, das einen Bezug zur Universität hat, ohne direkt als Forschungsdatenset oder wissenschaftliche Veröffentlichung eingeordnet werden zu können.

Im Bereich data information literacy bietet LTL Services an, die auch im Researcher Development Framework (RDF) Erwähnung finden. Beispielsweise wird die Relevanz des Forschungsdatenmanagements regelmäßig in Orientierungsprogrammen für Promovierende hervorgehoben, ebenso wie es Hinweise zu Open Acces und zur Open Data Policy der Universität gibt. Dies fällt in RDF in den Bereich A1 („Understands what constitutes robust and reliable data creation and the importance of good data management for analysis of information/ data“) und C1 (,Understands information/data ownership, and by extension the implications of copyright and licensing“) (Vitae 2012). Weitere Services und Materialien zur data information literacy müssten noch entwickelt werden.

\section{Fazit}

Abbildung 3 fasst die Problemstellungen zusammen, die sich für den neuseeländischen Bereich im März/April 2013 feststellen ließen. Sie beruht auf den Aussagen der Kolleg^innen in den Bibliotheken. Sie ist also zunächst nur als erster Hinweis zu sehen, welche Faktoren wichtig sind und müsste mit anderen Informationen, z. B. aus der universitären Ebene und anderen nationalen Kontexten, ergänzt werden, um sie zu einem allgemeingültigen Modell weiter zu entwickeln.

Deutlich wird jedoch auch jetzt schon, dass eine Förderpolitik, die den freien Zugang zu den Daten propagiert, wichtig ist, um Entwicklungen auf lokaler Ebene zu begleiten und zu befördern. In Deutschland geschieht dies bereits, wie die Förderpolitik der Deutschen Forschungsgemeinschaft zeigt. Auf lokaler Ebene gibt es ebenso bereits einige Universitäten, die eine institutional policy verabschiedet haben, zum Beispiel die Universität Bielefeld oder die Humboldt-Universität zu Berlin.

Der Ansatz in Lincoln ist sowohl auf theoretischpraktischer Ebene wie strukturell gesehen interessant, weil sich durch ein Team wie LTL Synergieeffekte generieren lassen, die für Services im Forschungsdatenmanagement gewinnbringend genutzt werden können. Allerdings ist die Universität Lincoln eine kleine Universität mit nur etwa 3500 Studierenden und 400 Forschenden. Es müsste also untersucht werden, ob das Modell für andere Rahmenbedingungen adaptiert werden könnte, beispielsweise für eine große Universität mit mehr Studierenden. Hier wäre denkbar, dass mittelfristig Teams für einzelne Fachbereiche gebildet werden, in denen neben Bibliothekar*innen auch die Forschenden beteiligt sind. An der Universitätsbibliothek selbst könnte es dann ein zentrales Team geben, das sowohl für die Infrastruktur als auch die inhaltliche Weiterbildung zum Beispiel der Fachreferent*innen zuständig ist und auf das die Fachbereichsteams zurückgreifen können. Eine ähnliche Struktur wurde an einer Universitätsbibliothek Neuseelands bereits erfolgreich eingeführt.

\section{Ausblick}

Nicht zuletzt in der Literatur wird immer wieder deutlich hervorgehoben, wie komplex und vielschichtig der Bereich Forschungsdatenmanagement ist. Für jeden lokalen Kontext dürfte sich die Situation zudem immer wieder etwas anders darstellen und Entwicklungen und Aktivitäten in diesem Bereich laufen bei weitem nicht linear und kontinuierlich in einer Richtung ab. Es wird zudem die Frage gestellt, ob sich wissenschaftliche Bibliotheken hier überhaupt engagieren sollten. Wie die Empfehlung der Hochschulrektorenkonferenz jedoch zeigt, geht es aber wohl nicht mehr um das Ob sondern das Wie. Sicherlich wird nicht jede Universitätsbibliothek eine umfangreiche technologische Infrastruktur aufbauen und eine große Bandbreite an Services im Forschungsdatenmanagement abdecken können noch müssen. Denn es geht nicht darum, das Rad an jedem Ort neu zu erfinden, sondern auf passende Services hinzuweisen, die es bereits in Deutschland, Europa oder sogar weltweit gibt. So verfolgt die Universität Lincoln nicht mehr uneingeschränkt den Ansatz, selbst ein großes Forschungsdatenrepositorium anzubieten, sondern verweist auch auf Angebote wie Figshare.

2 S. https://data.uni-bielefeld.de/de/policy und https://www.cms. hu-berlin.de/de/ueberblick/projekte/dataman/policy [15.06.2015] 
Gleichzeitig stellt sich die Frage, wie Bibliothekar*innen sich mit dem Forschungsdatenmanagement und den neuen Aufgaben vertraut machen können. Neben Materialien aus dem anglo-amerikanischen Bereich, wie sie z. B. im britischen Projekt MANTRA ${ }^{3}$ entwickelt und zur Verfügung gestellt wurden oder sie Cox et al. (2014) vorstellen, liefern insbesondere Erkenntnisse aus dem data information literacy-Projekt wertvolle Hinweise (Carlson et al. 2015).

Schließlich ist es hilfreich, sich zu vergegenwärtigen, dass das Thema Open Data für manche Disziplinen einen Paradigmenwechsel bedeutet und es hier Anknüpfungspunkte an die Argumentation im Bereich Open Access gibt: Die Grundlagen öffentlich geförderter Forschung sollen auch frei nachnutzbar sein. Ähnlich wie bei Open Access braucht es also einen langen Atem und Erfindungsreichtum. Aber es lohnt sich. Oder, wie vielleicht die Māori sagen würden: „Nā tō rourou, nā taku rourou ka ora ai te iwi.“4

\section{Literatur}

Büttner, S., Hobohm, H.-C., Müller, L. (Hrsg.) (2011): Handbuch Forschungsdatenmanagement. Bad Honnef: Bock + Herchen. URL: http://www.forschungsdatenmanagement.de [07.07.2015].

Bundy, Alan (Hrsg.) (2004): Australian and New Zealand Information Literacy Framework. 2. Auflage. Adelaide: Australian and New Zealand Institute for Information Literacy. URL: http:// archive.caul.edu.au/info-literacy/InfoLiteracyFramework.pdf [07.07.2015].

Carlson, J. et al. (2013): Developing an Approach for Data Management Education: A Report from the Data Information Literacy Project. In: International Journal of Digital Curation (1), S. 204-217. URL: http://www.ijdc.net/index.php/ijdc/article/ download/8.1.204/306 [07.07.2015].

Carlson, J., Johnston, L., Huang Y. (Hrsg.) (2015): Data Information Literacy. Librarians, Data and the Education of a New Generation of Researchers. Purdue Information Literacy Handbooks. Purdue University Press.

Corrall, S. (2012): Roles and Responsibilities: Libraries, Librarians and Data. In: Pryor, Graham (Hrsg.): Managing Research Data. London: Facet Publishing. S. 105-133.

Cox, M., Verbaan, E., Sen, B. (2014): A Spider, an Octopus, or an Animal Just Coming into Existence? Designing a Curriculum for Librarians to Support Research Data Management. In: Journal of eScience Librarianship 3(1), Article 2, URL: http://dx.doi. org/10.7191/jeslib.2014.1055 [07.07.2015]

3 S. http://datalib.edina.ac.uk/mantra/ [07.07.2015]

4 „With your food basket and my food basket the people will thrive.“ Frei übersetzt: „Mit deinem und meinem Essenskorb wird unsere Gemeinschaft aufblühen.“ (Kōrero Māori (o. J.): „Whakataukī - Proverbs“. URL: http://tetaurawhiri.govt.nz/learning-maori/tikangamaori/whakatauki-en-nz/ [07.07.2015].
Dawson, R. (2012): Making Visible the Experience and Activity in the Research Ecology at Lincoln University. An invited address to colleagues at the Holmes Bay Retreat, Banks Peninsula, 12 November 2012. Präsentation. URL: https://researcharchive. lincoln.ac.nz/handle/10182/5047 [07.07.2015].

Hochschulrektorenkonferenz (2012): Hochschule im Digitalen Zeitalter: Informationskompetenz neu begreifen - Prozesse anders steuern. Entschließung der 13. Mitgliederversammlung der HRK am 20. November 2012 in Göttingen. URL: http://www.hrk.de/ uploads/tx_szconvention/Entschliessung_Informationskompetenz_20112012_01.pdf [07.07.2015].

Nardi, B., O’Day, V. (1999): Information Ecologies. Cambridge, Massachusetts, London: MIT Press. Kapitel 4 „Information Ecology“ in: First Monday, 4 (5) 3 May 1999. URL: http://firstmonday.org/ojs/index.php/fm/article/view/672/582 [07.07.2015].

Pryor, Graham (Hrsg.) (2012): Managing Research Data. London: Facet Publishing.

SCONUL (2011): The SCONUL Seven Pillars of Information Literacy. A Research Lens for Higher Education. URL: http://www.sconul.ac.uk/sites/default/files/documents/researchlens.pdf [07.07.2015]. Stand: April 2011.

Steiner, K. (2013): Forschungsdatenmanagement und Informationskompetenz - Neue Entwicklungen in Hochschulbibliotheken Neuseelands. Masterarbeit. URN: urn:nbn:de:hbz:79pbc2014030638;

URL: http://publiscologne.fh-koeln.de/frontdoor/index/index/ docId/311 [07.07.2015].

Tappenbeck, I. (2015): Fachreferat 2020: from collections to connections. In: Bibliotheksdienst 49 (2015) 1, S. 37-48.

Vitae (2012): Information Literacy Lens on the Vitae Researcher Development Framework using the SCONUL Seven Pillars of Information Literacy. URL: https://www.vitae.ac.uk/vitaepublications/rdf-related/information-literacy-lens-on-the-vitaeresearcher-development-framework-rdf-apr-2012.pdf.

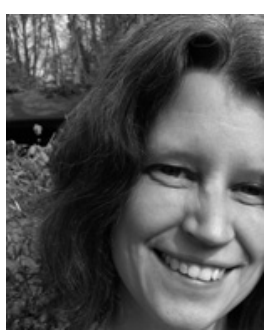

Katrin Steiner

LOTSE-Geschäftsstelle, ULB Informationskompetenz Universitäts- und Landesbibliothek Münster Krummer Timpen 3 48143 Münster Telefon 0251 83-25513 steinerk@uni-muenster.de

Katrin Steiner koordiniert seit 2008 die Aktivitäten im Bereich Informationskompetenz an der Universitäts- und Landesbibliothek Münster und hat die LOTSE-Geschäftsstelle inne. 2013 schloss sie den Studiengang Master of Library and Information Science an der FH Köln ab. 